\begin{tabular}{|c|c|c|}
\hline & $\begin{array}{l}\text { European Association for the } \\
\text { Development of Renewable Energies, Environment } \\
\text { and Power Quality (EA4EPQ) }\end{array}$ & $\begin{array}{l}\text { International Conference on Renewable Energies and Power Quality } \\
\text { (ICREPQ'12) } \\
\text { Santiago de Compostela (Spain), 28th to 30th March, } 2012\end{array}$ \\
\hline
\end{tabular}

\title{
Electrical losses in multi-MW wind energy conversion systems
}

\author{
A. Madariaga ${ }^{1}$, C. J. Martínez de Ilarduya ${ }^{1}$, S. Ceballos ${ }^{2}$, I. Martínez de Alegría ${ }^{1}$, J.L. Martín ${ }^{1}$ \\ ${ }^{1}$ Engineering Faculty of Bilbao, UPV/EHU University of the Basque Country \\ Alameda Urquijo s/n, 48013 Bilbao (Spain) \\ ander.madariaga@ehu.es, cjmartinezde001@hotmail.com, inigo.martinezdealegria@ehu.es, joseluis.martin@ehu.es \\ ${ }^{2}$ Tecnalia Research \& Innovation \\ Parque Tecnológico de Bizkaia C/Geldo, Edificio 700, 48160 Derio (Spain) \\ salvador.ceballos@tecnalia.es
}

\begin{abstract}
This paper proposes a procedure to assess the electrical losses in Wind Energy Conversion Systems (WECSs) with different electrical topologies. Those losses are evaluated in an annual basis considering the randomness of the wind with a Rayleigh probability distribution function. Due to the lack of specific standards, the analysis is based on standards applicable to low voltage (LV) and medium voltage (MV) installations. After reviewing the WECSs offered in main manufacturers' portfolio, three electric topologies have been studied with the proposed calculation procedure. The main difference among alternatives is the position of the power transformer and, therefore, the characteristics of the voltage in the wiring going down the tower. Another two alternative topologies with direct current (DC) output have also been studied. The parameters of the base case study are commented in detailed and the results of several sensitivity analyses are also presented.
\end{abstract}

\section{Key words}

Wind Energy Conversion Systems (WECSs), multi-MW, topologies, offshore, electric power losses, DC/DC transformer

\section{Introduction}

Wind power capacity installed offshore during the last year has been higher than expected. Nearly 900 MW of wind power capacity was added in 2010 , which puts the worldwide offshore installed wind capacity near 3.0 GW. This supposes a current growth rate for offshore wind power of about $50 \%$, clearly above the increase numbers in the onshore market for the same period, only slightly above $30 \%$ [1].

The size and rated power of wind turbines installed worldwide has increased continuously during the last decade. This has been particularly true in the design of wind turbines for offshore applications, as bigger turbines in conjunction with enlarged load factors and more windy sites compensate investors for the higher costs of offshore projects. A list of multi-MW wind turbines available in leading manufacturers' portfolio is presented in Table I. Windmills with a rated power up to $7.5 \mathrm{MW}$ are already available and even bigger platforms are currently under development [2].
Table I. - Available WECSs with rated power up to 7.0 MW.

\begin{tabular}{ccccc}
\hline Manufacturer & Model & $\begin{array}{c}\text { Power } \\
(\mathrm{MW})\end{array}$ & $\begin{array}{c}\text { Generator } \\
\text { type }\end{array}$ & $\begin{array}{c}\text { Gearbox } \\
\text { stages }\end{array}$ \\
\hline Enercon & E-126 & 7.5 & PMSG $^{1)}$ & 0 \\
Vestas & V164-7.0 & 7.0 & PMSG $^{2}$ & 2 \\
Repower & $6 \mathrm{M}$ & 6.2 & DFIG $^{2}$ & 3 \\
Repower & $5 \mathrm{M}$ & 5.0 & DFIG & 3 \\
Areva & M5000 & 5.0 & PMSG & 2 \\
Bard & VM & 5.0 & DFIG & 2 \\
Gamesa & G128-4.5 & 4.5 & PMSG & 2 \\
GE & $4.0-110$ & 4.0 & PMSG & 0 \\
Siemens & SWT-3.6 & 3.6 & SCIG & 3 \\
Vestas & V112/3.0 & 3.0 & PMSG & 4 \\
Vestas & V90/3.0 & 3.0 & DFIG & 3 \\
Winwind & WWD-3-110 & 3.0 & PMSG & 0 \\
Sinovel & SL 3.0/90 & 3.0 & DFIG & 3 \\
Siemens & SWT-2.3 & 2.3 & SCIG & 3 \\
\hline \multirow{5}{*}{ 1) PMSG } & Permanent Magnet Synchronous Generator & \\
2) DFIG & Doubly Fed Induction Generator & \\
3) SCIG & Squirrel Cage Induction Generator &
\end{tabular}

The electric systems of these multi-MW wind turbines present different topologies. For example, in Siemens' SWT-2.3-93 the generator is located in the nacelle and the converter-transformer are located at the base of the tower. In the SWT-3.6-107 the components of the power converter have been separated, with the rectifier installed in the nacelle and the inverter in the tower base. In Gamesa's G128-4.5 or in Vestas' V112-3.0, the LV/MV power transformer is placed in the nacelle and the electric power is taken down the tower in MVAC.

As we see, the optimum electrical topology continues to be an open issue, and the electrical losses in the cables inside the tower have become a major determinant with the increase in their rated power. Therefore, the location of the electronic converter and the power transformer is under consideration because it determines the type of the cables connecting the nacelle and the tower base [3].

With the analysis of those losses in mind, the rest of the paper is organized as follows. Section 2 presents the topologies considered in the study. The calculation procedure is detailed in section 3 . The results of a base case study and several sensitivity analyses are presented in section 4. Finally, section 5 concludes summarizing the key aspects of the paper. 


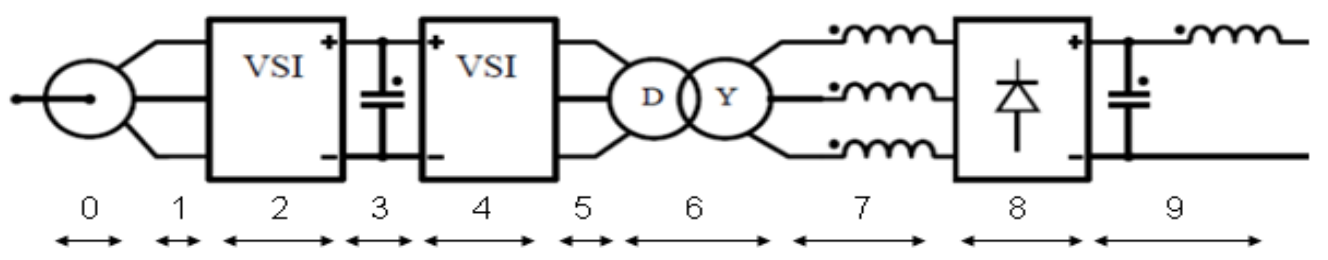

Figure 1. Scheme of the electrical installation of a generic wind turbine

\section{Considered alternatives}

We have considered five different WECS topologies in our calculation procedure. Three of these topologies give their electric power output in MVAC $(A, B$ and $C$ alternatives further on). These alternatives are based on topologies chosen by leading manufacturers for their multi-MW wind turbines and they have already been commented in the introduction (i.e. SWT-2.3 for the $A$ alternative, SWT-3.6 for the $B$ alternative, G128-4.5 and V112-3.0 for the $C$ alternative).

The other two alternatives give their electric power output in MVDC ( $B-D C$ and $C-D C$ alternatives further on). Although wind turbines with a DC output are not commercially available, they are being studied by the research community mainly due to the advantages of DC submarine transmission [4-6]. In these alternatives, the conventional power transformer working at industrial frequency has been substituted by a medium frequency transformer followed by a diode bridge rectifier. This transformer operates at $1 \mathrm{kHz}$ and its main advantages when compared with a conventional power transformer are a lower weight and size and that presents fewer losses than transformers operating at industrial frequency [7].

The main characteristics of the considered five alternatives are listed below.

A The back-to-back converter is placed close to the generator in the nacelle and the $50 \mathrm{~Hz}$ power transformer is located in the base of the turbine. The tower wiring operates at LVAC $(690 \mathrm{~V})$ and the output of the tower operates at MVAC $(33 \mathrm{kV})$.

$B \quad$ The back-to-back converter has the rectifier placed close to the generator in the nacelle and the inverter close to the power transformer in the tower base. The tower wiring operates at LVDC $(1100 \mathrm{~V})$ and the output of the tower operates at MVAC $(33 \mathrm{kV})$.

$B-D C$ As in the previous alternative, the tower wiring operates at LVDC $(1100 \mathrm{~V})$ but the output of the turbine operates at MVDC $(30 \mathrm{kV})$. The transformer operates at $1 \mathrm{kHz}$ and is placed in the tower base followed by a diode bridge rectifier.

$C \quad$ The $50 \mathrm{~Hz}$ power transformer is located up in the nacelle and, therefore, the tower wiring operates at MVAC (33 kV).

$C$-DC The $1 \mathrm{kHz}$ power transformer and the diode bridge rectifier are placed up in the nacelle and, therefore, the tower wiring operates at MVDC $(30 \mathrm{kV})$.

\section{Calculation procedure}

The description of the proposed calculation procedure is organized in four subsections. The first subsection introduces two key issues affecting the general structure of the calculation: the definition of a generic topology applicable to all the alternatives and the reasons for choosing the output power curve of a wind turbine as the main input for our calculation (as well as the implications this decision has on the calculation procedure). Then, the other three subsection focus on the assessment of the average energy yield, the power losses in wirings and the power losses in devices, respectively.

\section{A. General approach}

In general terms, the proposed calculation procedure takes the output power curve of the wind turbine as its input and estimates the electric power losses in each stage between the MV switchgears and the generator. To do so, a generic WECS is defined (figure 1). Each considered alternative $(A, B, C, B-D C$ and $C-D C)$ can be particularized from the generic topology by an appropriate election of the parameters that characterize each stage. The following list describes those stages and the parameters that characterize each stage are shown in brackets.

0 Generator. PMSG is considered. $\left(\mathrm{U}_{1}\right)$

1 LVAC wiring. Connects the generator and the electronic converter. $\left(l_{1}\right)$

2-4 Back-to-back electronic converter. The control of the inverter puts in its output a $50 \mathrm{~Hz}$ sinusoidal in $A, B$ and $C$ alternatives and a $1 \mathrm{kHz}$ square wave in $B-D C$ and $C$-DC alternatives. $\left(\mathrm{P}_{\text {rated }}{ }^{234}, \mathrm{p}_{\mathrm{NL}}{ }^{234}, \mathrm{p}_{\mathrm{FL}}{ }^{234}, \mathrm{U}_{3}, 1_{3}\right)$

5 LVAC wiring. It connects the back-to-back with the transformer. $\left(\mathrm{U}_{5}, \mathrm{l}_{5}\right)$

6 Power transformer. Different transformers are considered in the alternatives with a MVAC output or with a MVDC output. $\left(\mathrm{U}_{5} / \mathrm{U}_{7}, \mathrm{P}_{\text {rated }}{ }^{6}, \mathrm{p}_{\mathrm{FL}}{ }^{6}, \mathrm{p}_{\mathrm{FL}}{ }^{6}\right)$

7 MVAC wiring. In $A, B$ and $C$ alternatives it links the $50 \mathrm{~Hz}$ transformer with the MV switchgear. In $B-D C$ and $C-D C$ alternatives, connects the medium frequency transformer with the diode bridge. $\left(l_{7}\right)$

8 Diode bridge rectifier. It is exclusive of the MVDC alternatives $(B-D C$ and $C-D C) .\left(\mathrm{P}_{\text {rated }}^{8}, \mathrm{p}_{\mathrm{NL}}^{8}, \mathrm{p}_{\mathrm{FL}}{ }^{8}\right)$

9 MVDC Wiring $(30 \mathrm{kV})$. It is exclusive of the MVDC alternatives. It links the rectifier with the MV switchgear. $\left(\mathrm{U}_{9}, \mathrm{l}_{9}\right)$ 
The consideration of the power curve of the turbine as the input for the calculation, demands an iterative procedure to determine the losses. The power input to stage 1 has to be adjusted to obtain the value given by the power for the correspondent operating range. This output is given in MVAC or MVDC depending on the alternative considered.

A direct approach (i.e. starting with the power captured from the wind in the rotor's shaft and calculating the losses from there on) demands a detailed characterization of the wind's energy capture of the turbine and also of the losses in all the stages from there on. For example, the model of wind turbine used in the procedure described in [8] demands a considerable number of parameters which are not usually disclosed by wind turbine manufacturers. On the contrary, the output power curve of a wind turbine is usually in datasheets disclosed by manufacturers.

\section{B. Average Energy Yield}

The operating range of the wind turbine is binnarized in 1 $\mathrm{m} / \mathrm{s}$ steps. As the G128-4.5 wind turbine has a cut-in wind speed of $3 \mathrm{~m} / \mathrm{s}$, reaches its rated output power near $12 \mathrm{~m} / \mathrm{s}$ and has a cut-out wind speed of $25 \mathrm{~m} / \mathrm{s}$ we have ten operating ranges and another non-operating range for speeds below cut-in or above cut-out. The power output of the wind turbine in each operating range is taken from its power curve. In the non operating range, we consider that the power transformer has to be maintained energized in order to supply energy for auxiliary services (yaw control, brake system, telecommunications, nacelle air conditioning, etc.). We assess the power consumption in the non operating range by the no-load losses of the transformer and of the electronic converters.

We estimate for how many hours a year the wind blows in a certain speed range by the Rayleigh probability density function [9]. We will use the equation (1) for the calculation of the cumulative probability (CP) for a given wind speed $\left(\omega_{\mathrm{s}}\right)$.

$$
C P=1-e^{-\left(\omega_{s} / c\right)^{2}}
$$

The $c$ parameter, which describes the sharpness of the distribution, is calculated from the average wind speed at the site $\left(v_{a}\right)$ with equation (2).

$$
v_{a}=\frac{c^{*} \sqrt{\pi}}{2}
$$

The number of hours that the wind speed blows in each of the considered speed ranges is determined multiplying the value of their cumulative probability with the number of operating hours in a year.

Finally, the energy yield of the wind turbine can be determined in each operating range multiplying the power output in that range by the number of hours a year the turbine is expected to function in that speed range. Adding the energy yields in all the operating ranges we get the average energy yield in an annual basis.

The power losses are calculated separately for each of the eleven power ranges. Those calculations follow the procedures for the electric power losses in wirings and devices described in the next subsections. Those losses are averaged also in an annual basis with the procedure described for the energy production.

It should be remarked that the non-operating range will have a negative yield, as corresponds to the energy consumption for auxiliary services. But the electric power losses will also be present in that case and the operator of the wind farm will have to pay for them.

\section{Electric power losses in wirings}

For stretches operating in $\mathrm{AC}\left(1^{\text {st }}, 5^{\text {th }}\right.$ and $\left.7^{\text {th }}\right)$, we start calculating the power input to the analysed wiring stretch subtracting the losses originated in the previous one. Then, the circulating intensity is calculated with equation (3), which relates the electric active power $(P)$ with the intensity $(I)$, the line-to-line voltage $(U)$ and the power factor $(\cos \varphi)$.

$$
\mathrm{P}=\sqrt{3} * \mathrm{U}^{*} \mathrm{I}^{*} \cos \varphi
$$

For the stretches operating in DC $\left(3^{\text {rd }}\right.$ and $\left.9^{\text {th }}\right)$, we follow a similar procedure but applying equation (4), which relates the electric power $(P)$ with the DC voltage $(V)$ and current $(I)$.

$$
\mathrm{P}=\mathrm{V}^{*} \mathrm{I}
$$

Following the criteria of thermal dimensioning, the maximum intensity value determines the number of conductors necessary in each stretch. Consequently, when the wind turbine does not work at its rated power those wirings will be oversized.

The number of conductors will be determined according to [10] for stretches operating in $\operatorname{LV}\left(1^{\text {st }}, 3^{\text {rd }}\right.$ and $\left.5^{\text {th }}\right)$. For wirings operating in $\mathrm{MV}\left(7^{\text {th }}\right.$ and $\left.9^{\text {th }}\right)$ the recommendations found in [11] will be followed. Correction factors must be established according to the operating conditions of the installation and the maximum permissible intensity which depends on the section of the conductors. For the stretches operating in DC $\left(3^{\text {rd }}\right.$ and $\left.9^{\text {th }}\right)$ it has been considered that the direct current value matches the rms value in the aforementioned regulations. Hence, the application of the tabulated values is direct.

The temperature reached by the conductor $(T)$ in each operating range is calculated with equation (5), where the actual current $(I)$, the maximum permissible current $\left(I_{\text {perm }}\right)$, the temperature of the environment $\left(T_{\text {env }}\right)$ and the maximum temperature the wire can reach to prevent damage to its isolation $\left(T_{\max }\right)$ must be indicated [12].

$$
\mathrm{T}=\mathrm{T}_{\text {env }}+\left(\mathrm{T}_{\text {máx }}-\mathrm{T}_{\text {env }}\right) *\left(\frac{I}{I_{\text {perm }}}\right)^{2}
$$

The DC resistance value at operating temperature $\left(\mathrm{R}_{\mathrm{DC}}{ }^{\mathrm{T}}\right)$ is calculated with equation (6) [13]. The DC resistance value at $20^{\circ} \mathrm{C}$ is taken from [14] and the coefficient of variation of resistivity with temperature $(\alpha)$ is taken from [13]. 


$$
\mathrm{R}_{\mathrm{DC}}^{\mathrm{T}}=\mathrm{R}_{\mathrm{DC}}^{20^{\circ} \mathrm{C}} *\left[1+\alpha^{*}(\mathrm{~T}-20)\right]
$$

The AC resistance for each speed range $\left(R_{A C}{ }^{T}\right)$ is calculated with equation (7) which follows the indications for the skin effect factor $\left(y_{s}\right)$ and the proximity factor $\left(y_{p}\right)$ given in [13]. In the event of a breach of any imposed condition in the mentioned standard, [15] provides an alternative procedure for the calculation of $R_{A C}{ }^{T}$. Contrasting both procedures for wirings operating at 1 $\mathrm{kHz}$, where the results of equation (7) are slightly out its scope according to [13], we have seen great coincidence.

$$
\mathrm{R}_{\mathrm{AC}}^{\mathrm{T}}=\mathrm{R}_{\mathrm{DC}}^{\mathrm{T}} *\left(1+\mathrm{y}_{\mathrm{s}}+\mathrm{y}_{\mathrm{p}}\right)
$$

Following recommendations given in [13], the losses in screens of MVAC cables are estimated with equation (8)

$$
\lambda_{1}=\lambda_{1}^{\prime}+\lambda_{1}^{\prime \prime}
$$

where $\lambda_{1}$ indicates the total losses in the screen over the total losses in the conductors, $\lambda_{1}{ }^{\prime}$ the losses in the screen produced by the circulating currents and $\lambda_{1} "$ the losses generated by the Foucault currents.

In order to assess the losses in the screen, the procedure described in [13] demands the resistance of the screen and, therefore, its operating temperature has to be estimated. In the absence of thermal conductivity data on the layers of the wire (that could allow estimation based on Fourier's law), it has been considered that the difference of temperature between the conductor and the environment decreases by two-thirds of its value in the isolation and a third in the cover. This decision is based on the approximated ratio of the thickness of the layers that form the wire [16] and in the assumption that screen temperature is the same in all its points. This assumption seems reasonable because the screen is metallic (usually $\mathrm{Cu}$ ) and does not offer much opposition to the transmission of heat.

This procedure for the calculation of losses in the screen based in [13] is only valid for $50 \mathrm{~Hz}$. However, in B-DC and $\mathrm{C}$-DC alternatives stage 7 is working at $1 \mathrm{kHz}$. In those cases we have assessed the losses at $1 \mathrm{kHz}\left(\lambda_{l}^{f}\right)$ with equation (9), which considers the losses at $50 \mathrm{~Hz}\left(\lambda_{I}{ }^{50}\right)$ and the relation of the resistances at both frequencies $\left(R s_{A C} f\right.$ and $R s_{A C}{ }^{50}$ ).

$$
\lambda_{1}^{\mathrm{f}}=\lambda_{1}^{50} * \frac{\mathrm{Rs}_{\mathrm{AC}}^{\mathrm{f}}}{\mathrm{Rs}_{\mathrm{AC}}^{50}}
$$

Finally, electrical power losses $(P)$ are calculated in each operating range using equations (10-12), where $L$ stands for for the length of the considered wiring. Equation (10) is used for $3^{\text {rd }}$ and $9^{\text {th }}$ stretches, equation (11) for $1^{\text {st }}$ and $5^{\text {th }}$ and equation (12) for the $7^{\text {th }}$ stretch.

$$
\begin{aligned}
& \mathrm{P}=\mathrm{L}^{*} \mathrm{I}^{2} *\left(\mathrm{R}_{\mathrm{DC}}^{\mathrm{T}} / \text { circuits }\right) \\
& \mathrm{P}=\mathrm{L}^{*} 3 * \mathrm{I}^{2} *\left(\mathrm{R}_{\mathrm{AC}}^{\mathrm{T}} / \text { circuits }\right) \\
& \mathrm{P}=\mathrm{L}^{*} 3 * \mathrm{I}^{2} *\left[\mathrm{R}_{\mathrm{AC}}^{\mathrm{T}} *\left(1+\lambda_{1}\right) / \text { circuits }\right]
\end{aligned}
$$

\section{Electric power losses in devices}

In this subsection we detail how electric power losses are assessed in the back-to-back electronic converter $\left(2^{\text {nd }}\right.$ and $4^{\text {th }}$ devices $)$, in the power transformer $\left(6^{\text {th }}\right.$ device $)$ and in the diode bridge rectifier $\left(8^{\text {th }}\right.$ device in $B-D C$ and $C-D C$ alternatives). In all of them, we considered the variation of electric losses linear with power input $\left(\mathrm{P}_{\text {input }}^{\mathrm{i}}\right)$ and characterized by the rated power of the device $\left(\mathrm{P}_{\text {rated }}^{\mathrm{i}}\right)$, the no-load losses percentage $\left(\mathrm{p}_{\mathrm{NL}}^{\mathrm{i}}\right)$ and the full-load losses percentage $\left(p_{F L}^{i}\right)$. Equation (13) shows the formulation used in the assessment of those losses.

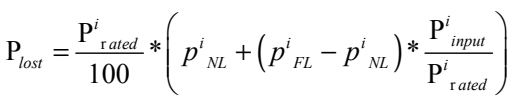

Regarding the losses in the back-to-back, this assumption is supported in data from the electronic converter mounted in the G128-4.5 wind turbine [17-18]. The linearity is clear in figure 2, and these data have been used further on to characterize the losses in the converter.

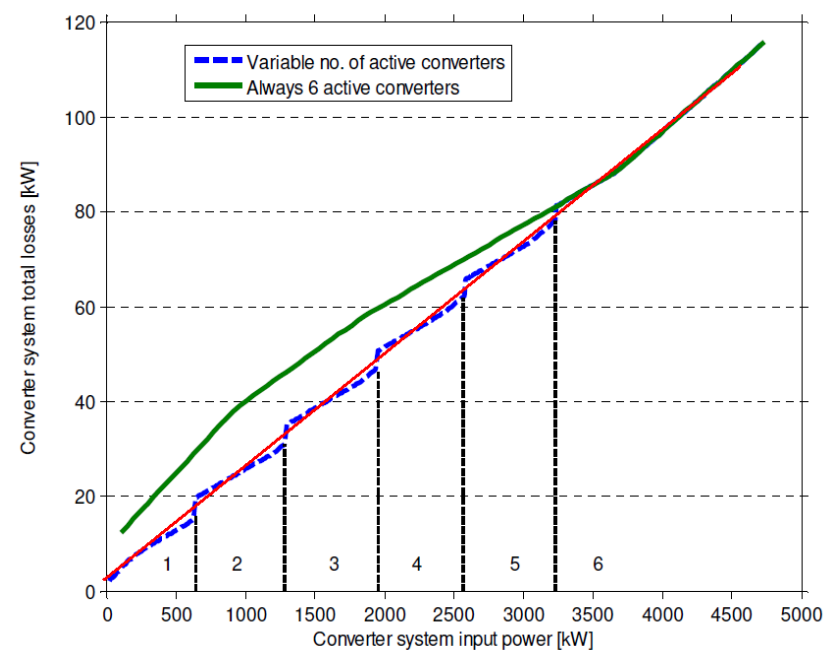

Figure 2. Power losses in G128's actively modular back-to-back

For transformers and diode bridge rectifiers, the linearity assumption and the parameters considered in the base case study are supported in $[4,5,19,20]$.

\section{Results}

\section{A. Base Case Study}

The proposed calculation procedure has been applied to a base case study with the following parameters:

- Wind turbine rated power: $4.5 \mathrm{MW}$

- Tower height: $100 \mathrm{~m}$

- Transformer frequency: $50 \mathrm{~Hz}$ and $1 \mathrm{kHz}$ in MVAC and MVDC alternatives respectively.

- Power factor of wind turbine: $\cos \varphi=1$

- Temperatures: $20^{\circ} \mathrm{C}$ inside the nacelle and $40^{\circ} \mathrm{C}$ inside the tower.

- Coefficient device sizing: 1.33

- Generator output voltage: $690 \mathrm{~V}$

- Back-to-back:

○ Losses: $0.07 \%$ no load and $2.4 \%$ full load

- DC bus voltage: $1100 \mathrm{~V}$

○ Output voltage: $690 \mathrm{~V}$ 
- Transformer: $6 \mathrm{MW}$ rated power and $0.69 / 33 \mathrm{kV}$ transformation ratio.

○ Losses: $0.2 \%$ no load and $0.6 \%$ full load for $50 \mathrm{~Hz}$ and $0.07 \%$ no load and $0.153 \%$ full load for $1 \mathrm{kHz}$.

- Rectifier: $6 \mathrm{MW}$ rated power, $30 \mathrm{kV}$ output voltage.

○ Losses: $0 \%$ no load and $0.33 \%$ full load.

The results of applying the procedure of the calculation to the base case study are shown in the Table II. From them, it can be concluded that the alternatives which have a lower percentage of losses are the ones from group C, followed by the ones from group B and finally, alternative A. It is remarkable that typologies with MVDC output have lower percentages of losses than the ones with MVAC output. In order to establish the cost of those losses, the energy transferred to the grid has been given a value of $80 \mathrm{EUR} / \mathrm{MWh}$ (operating ranges) and the cost of the energy demanded has been set in $160 \mathrm{EUR} / \mathrm{MWh}$ (nonoperating range).

Table II. - Electric power losses in each alternative.

\begin{tabular}{cccc}
\hline Losses & $\%$ & MWh/year & EUR/year \\
\hline A & 4.41 & 689 & 56854 \\
B & 4.03 & 628 & 51991 \\
B-DC & 3.60 & 568 & 46061 \\
C & 3.68 & 573 & 47590 \\
C-DC & 3.27 & 515 & 41802 \\
\hline
\end{tabular}

\section{B. Sensitivity Studies}

To analyse how the variation of the input parameters could affect the results presented, several sensitivity analysis have been performed (Fig. 3).

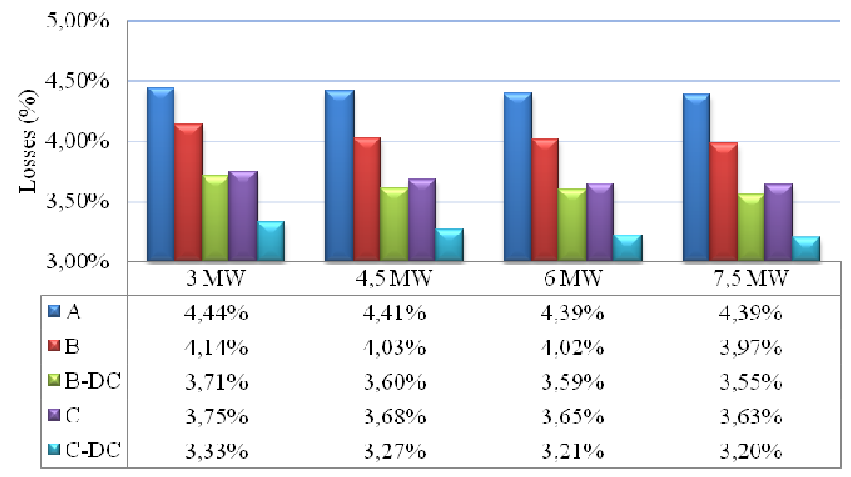

(a) Influence of rated power.

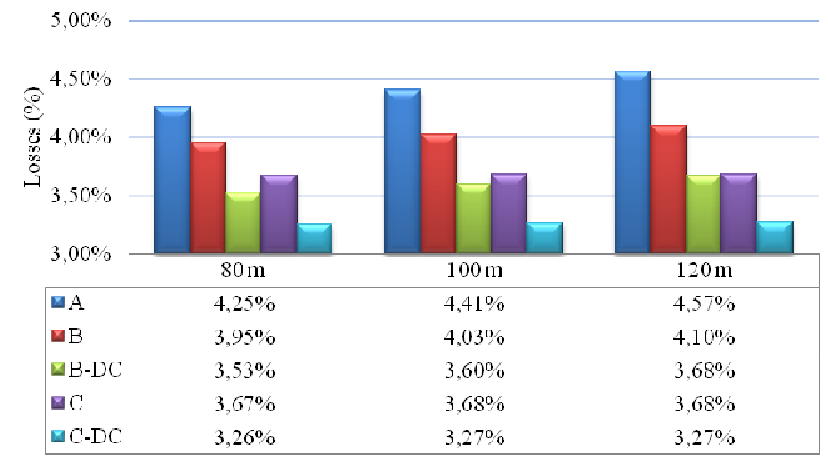

(c) Influence of the height of the tower.
Following the suggested calculation procedure, we have analysed the cases listed below.

Rated power: 3, 4.5, 6 and 7.5 MW.

Wind speed: $6,7,8,9,10,11$ and $12 \mathrm{~m} / \mathrm{s}$.

Tower height: 80, 90, 100, 110 and $120 \mathrm{~m}$.

Temperature inside the tower: 20,40 and $60^{\circ} \mathrm{C}$.

Power factor: $1,0.95$ and 0.9 .

Regarding rated power of the wind turbine, it is found that the average percentage of losses decreases with the increase of the rated power (figure 3.a). Although logically losses increase in absolute terms, this effect is because of a greater increase of the energy production with the rated power than that of the losses.

Considering the variation of the average wind speed at the site, it is found that the percentage of losses also decreases with the increase in the mean wind in all typologies (figure 3.b). We also notice that the alternatives with MV wirings inside the towers are favoured with more windy sites, for example when we compare $B$ with $C$ or $B-D C$ with $C-D C$.

Regarding the height of the tower, figure 3.c shows that in alternatives A, B and B-DC the percentage of losses increases faster with the increase of the height than in alternatives $\mathrm{C}$ and C-DC. On the other hand, figure 3.d shows that the influence of the variation of the operating temperature inside the tower can be neglected in further studies.

Although no figure has been included, alternative $\mathrm{A}$ is most affected by a change in the power factor as it could be expected. All in all, sensitivity studies confirm the conclusions got from the results of the base case study.

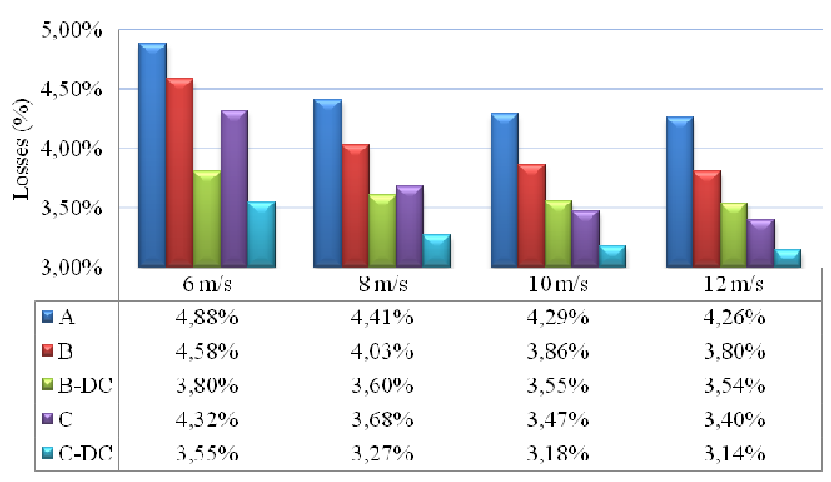

(b) Influence of the mean wind speed.

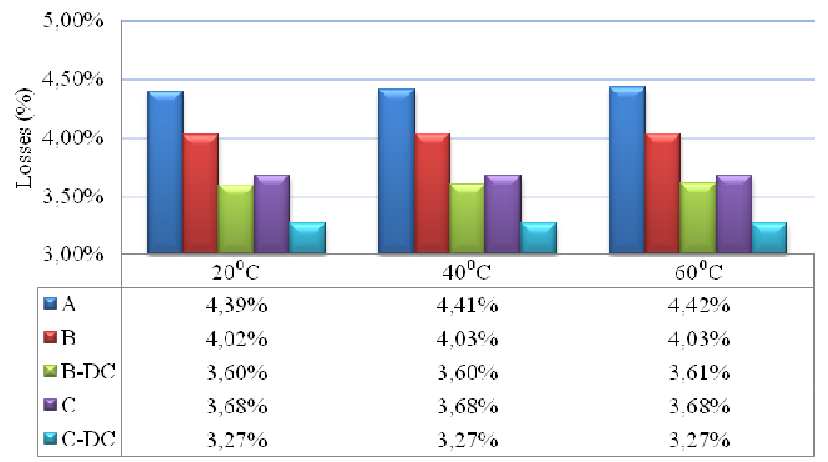

(d) Influence of the temperature inside the tower.

Figure 3. Results of sensitivity analysis 


\section{Conclusion}

A procedure for the assessment of the average electric power losses in WECSs with different topologies has been presented in detail. It is based on standards applicable to similar installations, on information about industrial cables and converters and on academic studies presented during the last decade. Theoretical WECS topologies with power output in MVDC have also been considered when developing the procedure.

The base case studied with the proposed methodology quantifies the difference in terms of electric power losses between different alternatives. The alternatives with LV wiring in their towers show a poor performance, which goes in line with the trends in new multi-MW wind turbines. The alternatives with MV wiring in their towers show certain advantage when compared with those in which the power is taken down the tower with the DC bus of the back-to-back converter. However, as the location of the power transformer influences the weight of the nacelle and, therefore, the strength and cost of the tower both influences should be counterbalanced in a final decision.

The sensitivity analyses performed confirm the conclusions mentioned in the previous paragraph. They also show that the advantage of alternatives with MV wirings in their tower increases with the increase in the rated power of those wind turbines, with the increase of the mean wind at the site and also with the height of the tower.

\section{Acknowledgements}

The investigation has been accomplished in the GAMESA Classroom at the Engineering Faculty of Bilbao, University of the Basque Country, with the collaboration of Tecnalia Research \& Innovation.

\section{References}

[1] A. Madariaga, I. Martínez de Alegría, J.L. Martín, P. Eguía and S. Ceballos. "Market forecasts, feasibility studies and regulatory framework for offshore wind energy integration". IECON 2011 proceedings, Melbourne, November 2011.

[2] Windpower. "Wind turbines and wind farms database". Available at: http://www.thewindpower.net/index.php. (Retrieved on October 2011)

[3] A. Madariaga, I. Martínez de Alegría, J.L. Martín, P. Eguía and S. Ceballos. "Analysis of the technology currently used in offshore wind energy systems". IECON 2011 proceedings, Melbourne, November 2011.

[4] S. Lundberg. "Performance comparison of wind park configurations". Department of Electric Power Engineering, Chalmers University of Technology, Technical Report, 2003.

[5] C. Meyer, M. Hoing, A. Peterson, and R. W. De Doncker. "Control and design of DC grids for offshore wind farms". IEEE Transactions on Industry Applications, vol. 43, no. 6 , pp. 1475-1482, 2007.

[6] I. Martínez de Alegría, J.L. Martin, J. Andreu, H. Camblong and P. Ibanez. "Tapping wind turbines to HVDC lines” EPE 2009 proceedings, Barcelona, September 2009.
[7] I. Martínez de Alegría, A. Madariaga, J.L. Martín, H. Camblong and S. Ceballos. "Effect of overlap in medium voltage high power high frequency transformers". PCIM Europe 2011 proceedings, Nuremberg, May 2011.

[8] O. Gomis-Bellmunt, A. Junyent-Ferre, A. Sumper, and S. Galceran-Arellano. "Maximum generation power evaluation of variable frequency offshore wind farms when connected to a single power converter". Applied Energy, vol. 87, no. 10, pp. 3103-3109, October 2010.

[9] R.J. Barthelmie, S.C. Pryor and S.T. Frandsen. "Climatological and Meteorological Aspects of Predicting Offshore Wind Energy (Offshore Wind Power)". G. Gaudiosi and J. Twidell, Eds. Multi-Science Publishing Co. Ltd, 2009.

[10] UNE 20460-5-523. "Electrical installations of buildings. Part 5: Selection and erection of electrical equipment. Section 523: Current-carrying capacities in wiring systems". (It adopts the International Standard IEC 60364-5-523:1999)

[11] UNE 211435. "Guidance on the selection of distribution cables for rated voltages up to and including $0.6 / 1.0 \mathrm{kV}$ ". December 2007.

[12] FLEXIGRON 1000 DN-F 0.6/1.0 kV Datasheet. General Cable.

[13] UNE 21144-1. "Electric cables. Calculation of the current rating. Part 1: Current rating equations (100\% load factor) and calculation of losses." September 2002. (It adopts the International Standard IEC 60287-1:1994)

[14] UNE-EN 60228. "Conductors of isolated cables". November 2004. (It adopts the International Standard IEC 60228)

[15] R.A. Chipman. "Theory and calculation problems in transmission lines”. Ed. McGraw-Hill, Panamá, 1971.

[16] HERSATENE RHZ1 H-16 Al 18/30 kV Datasheet. General Cable.

[17] B. Andresen and J. Birk. "A high power density converter system for the Gamesa G10X 4.5 MW wind turbine". In IEEE Power Electronics and Applications European Conference, September 2007.

[18] J. Birk and B. Andresen. "Parallel-connected converters for optimizing efficiency, reliability and grid harmonics in a wind turbine". In IEEE Power Electronics and Applications European Conference, September 2007.

[19] S. Meier and P.C. Kjaer, "Benchmark of annual energy production for different wind farm topologies", in $36^{\text {th }}$ Annual IEEE Power Electronic Specialists Conference (PESC 05). IEEE, June 2005, pp. 2073-2080.

[20] P. Bresesti, W. L. Kling, R. L. Hendriks and R. Vailati. "HVDC connection of offshore wind farms to the transmission system". IEEE Transactions On Energy Conversion, vol. 22, no. 1, pp. 37-43, 2007 